\title{
A statistical analysis of subsampling and an evaluation of the Folsom plankton splitter
}

\author{
Daniel W. Sell \& Marlene S. Evans \\ Great Lakes Research Division, University of Michigan, Ann Arbor, MI 48109, U.S.A.
}

Keywords: subsampling, Folsom splitter, zooplankton, Great Lakes, survey design

\begin{abstract}
Subsampling techniques are important for the determination of precise plankton density estimates. A binomial model of random subsampling, and its Poisson extension, were developed for the purpose of evaluating the performance of compartment-type plankton subsamplers. Two approaches were used to assess the performance of the Folsom plankton splitter on an extensive series of nearshore Lake Michigan crustacean zooplankton samples collected between 1974 and 1979. First, Folsom subsamples were observed to be significantly $(\mathrm{p}<0.05)$ more variable than expected from the rand om model of subsampling. Second, a random effects ANOVA model was used to compare fractions of the total variance in density estimates that were attributable to subsampling and sampling phases of a specially designed study. Departures from randomness in subsampling were sufficiently small that an analysis of optimal allocation of effort between subsampling and sampling phases, based on the ANOVA model, indicated that only one to three subsamples needed to be examined per sample.
\end{abstract}

\section{Introduction}

Subsampling of plankton samples is usualy necessary because of the large numbers of organisms caught with net hauls, water bottles, plankton traps and other collecting techniques. In an excellent review of statistical aspects of plankton sampling, Cassie (1971) categorized subsamplers into three functional types: 1) subsamplers having compartments into which the sample is divided (e.g. the Folsom splitter), 2) pipette subsamplers (e.g. the Hensen-Stempel pipette), and 3) chambers in which the subsample is part of a larger field (e.g. the Sedgwick-Rafter cell). Several compartment-type subsamplers(Gibbons 1933; Wiborg 1951; McEwen et al. 1954; Motoda 1959; Cushing 1961; Mednikov \& Starobogatov 1961; Waters 1969; Hickley 1975) have been widely used for a variety of freshwater and marine organisms. Modifications of the Folsom splitter (Longhurst \& Seibert 1967; Scarola \&
Novotny 1968) and the Whirling vessel (Kott 1953) have been proposed to aid in the ease and reliability of the use of these devices.

Two statistical questions should be posed when evaluating the utility of a subsampling device for any kind of samples. First, are estimates derived from the use of the subsampler statistically unbiased? Second, how large is the variability in estimates resulting from subsampling? Cassie (1971) generalized that compartment subsamplers are superior to pipette devices in the ease and certainty with which bias can be eliminated, while chambers have intermediate characteristics. The second question is important in evaluating what size subsamples should be (i.e. how many organisms should be enumerated in a subsample) and how many replicate samples versus how many replicate subsamples should be examined in a particular study.

Ricker (1937) suggested the use of binomial models to describe the variability inherent in the

Hydrobiologia 94, 223-230 (1982). 0018-8158/82/0943-0223/\$01.60. (c) Dr W. Junk Publishers, The Hague. Printed in The Netherlands. 
plankton subsampling process. McEwen et al. (1954) evaluated a complicated model, which included a binomial component, for subsampling marine zooplankton using a Folsom plankton splitter. Ricker's simple binomial model, and its Poisson extension, will be developed here to examine the bias and variability associated with the operation of compartment-type subsamplers. The development of these models clarifies the notion of 'randomness' of subsamples and allows a straightforward assessment of subsamplers. Further, analysis of variance is used to estimate variability introduced by subsampling in the context of variability in collecting replicate vertical net hauls at a location in Lake Michigan. These variance component estimates are used to derive an optimal allocation of effort between these sampling phases for this study. Although we assess the randomness of subsampling Great Lakes crustacean zooplankton with a Folsom plankton splitter, the statistical procedures have general use. Evaluations of laboratory subsampling techniques are an important aspect of the design of surveillance, monitoring, and other quantitative studies.

\section{Materials and methods}

As part of an environmental monitoring program for the Donald C. Cook Nuclear Power Plant, our group has been collecting zooplankton samples in southeastern Lake Michigan since 1970. Station locations, collection procedures, and laboratory techniques used for these samples are described by Evans(1975), Evans et al. (1978), Hawkins \& Evans (1979), and Evans et al. (1980). Folsom plankton splitters have been used for subsampling in our program since 1972. Plankton in the Folsom splitter were stirred vigorously with a glass stirring rod to disperse clumps. The operator judged when plankton were best dispersed within the splitter before dividing the sample (Longhurst \& Seibert 1967). The Folsom subsampler was examined daily or weekly to ensure it was level by measuring volumes of water split into aliquots. The Folsom splitter was maintained so that it divided a $500 \mathrm{ml}$ volume of water into $250 \pm 2 \mathrm{ml}$ aliquots. Typically, a sample fraction was selected so that 350-700 animals could be enumerated in each of two paired subsamples obtained from a sample.
A special series of collections was taken on 13 June 1980 for a study of analysis of variance (ANOVA). Fourteen replicate vertical net hauls were made from $2 \mathrm{~m}$ above the bottom to the surface at a 52-m station in Lake Michigan directly west of St. Joseph, Michigan. The first seven hauls employed a $0.5 \mathrm{~m}$ diameter $78-\mu \mathrm{m}$ mesh net and the last seven hauls were taken with a $0.5 \mathrm{~m}$ diameter $156-\mu \mathrm{m}$ mesh net. Sampling was conducted between 1615 and 1820 EDT. Calibrated flowmeters were used in the mouth of each net. In the laboratory, these samples were also subsampled with a Folsom plankton splitter, but the subsampling procedure differed from the routine samples. Preliminary examination of the samples indicated a high degree of subsampling was required. A subsampling scheme was set up to allow four replicate subsamples of a 1/ 1024 fraction to be randomly selected for examination from each sample. The 1024 possible subsamples that could be generated with 10 sequential uses of the splitter were ordered and numbered (Fig. 1). Four random numbers between 1 and 1024 were drawn from a random number table to determine which subsamples would be examined from each sample. A one-way random effects ANOVA model was used to estimate variance components due to subsampling (4 replicates) and vertical hauls ( 7 replicates) for the data from each mesh netting.

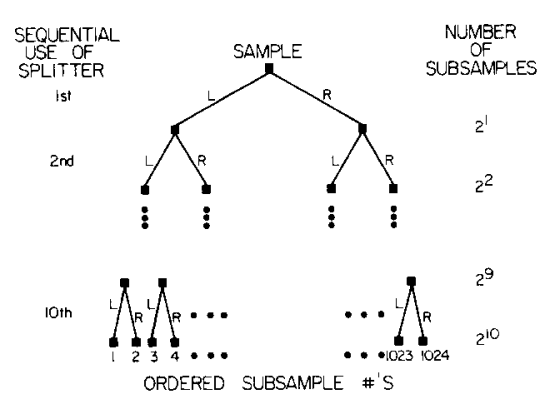

Fig. I. Schematic illustration of the subsampling design to generate replicate subsamples of $\mathrm{I} / 1024$ fraction from ten sequential uses of the Folsom plankton splitter. The numbering of the resulting subsamples is shown. Note that subsample \#1 is generated by ten sequential uses of the splitter in which the left aliquot is always chosen for further subdividing. 


\section{Results}

\section{General subsampling model}

Most subsampling devices are designed so that each compartment should receive an equal fraction of the sample to be divided. Thus, the expected value of the fraction of the sample settling into the $\mathrm{i}$-th compartment $\left(\mathrm{E}\left(\mathrm{p}_{\mathrm{i}}\right)\right)$ is usually assumed to be $E\left(p_{i}\right)=1 / I$, where $I$ is the number of compartments in the device. Several studies have examined the utility of subsampling devices for various types of organisms by statistically testing whether $\mathrm{E}\left(\mathrm{p}_{\mathrm{i}}\right)=$ $1 /$ I for all I compartments (Scarola \& Novotny 1968; McWilliam 1970). If it is known that $E\left(p_{i}\right)=$ $1 / \mathrm{I}$, the total number of animals in the sample $(Y)$ can be estimated $(\hat{Y})$ from a count of the animals in the $\mathrm{i}$-th compartment $\left(\mathrm{y}_{\mathrm{i}}\right)$ :

$\hat{\mathrm{Y}}=\mathrm{I} \mathrm{y}_{\mathrm{i}}$.

Also, it can be shown that $\hat{Y}$ is an unbiased estimator of $Y$, i.e. that the expected value of $\hat{Y}, E(\hat{Y})$, is equal to $Y$. Even if the expected fractions of animals falling into the different compartments are unequal and unknown, it can be shown that $E\left(p_{i}\right)=1 / I$ and $\mathrm{E}(\hat{\mathrm{Y}})=\mathrm{Y}$ provided the subsample counted is selected randomly (Cassie 1971), with an equal probability of selecting any of the I subsamples. However, there is still an important advantage in designing subsampling devices so that all compartments should receive equal fractions of a sample. When selection of one of I subsamples is randomly determined, the variance of counts of animals in subsamples selected in this manner is minimized when $E\left(p_{i}\right)=1 /$ I for all compartments.

The subsampling scheme may be extended by taking subsamples from subsamples. Let $y_{i j}$ be the number of animals settling into the $j$-th compartment upon re-splitting the i-th subsample. Furthermore, let $p_{j}=y_{i j} / y_{i}$, and let $p_{i j}=y_{i j} / Y$. Assuming that a second use of the subsampler is independent of the first, then

$E\left(p_{i j}\right)=E\left(p_{j} p_{i}\right)=E\left(p_{j}\right) E\left(p_{i}\right)=(l / I)^{2}$

$\hat{\mathrm{Y}}=\mathrm{I}^{2} \mathrm{y}_{\mathrm{ij}}$

and it can be shown that $\hat{Y}$ is an unbiased estimator of $Y$. This general scheme may be extended to any number of sequential subsamples, assuming that each use of the subsampler is independent of previous uses. The assumption of independence may simply be met by randomly choosing the subsample to be further subdivided.

The variance of the estimate of total number, $\mathrm{V}(\hat{\mathrm{Y}})$, may be determined from survey sampling methods (Raj 1968; Sukhatme \& Sukhatme 1970) by counting the number of animals in replicate subsamples and estimating $\mathrm{V}\left(\mathrm{y}_{\mathrm{k}}\right)$ directly:

$\hat{\mathrm{V}}(\hat{\mathrm{Y}})=\hat{\mathrm{V}}\left(\dot{\mathrm{y}}_{\mathrm{k}}\right) / \mathrm{E}\left(\mathrm{p}_{\mathrm{k}}\right)^{2}$

where $y_{k}$ is, in the general case, the number of animals in the $p_{k}$ sample fraction. If the estimate of $\hat{\mathrm{Y}}$ is based on the mean number of animals $\left(\overline{\mathrm{y}}_{\mathrm{k}}\right)$ in $\mathrm{n}$ of $\mathrm{N}$ (where $\mathrm{N}=1 / \mathrm{p}_{\mathrm{k}}$ ) possible replicate subsamples, then

$\hat{\mathrm{Y}}=\mathrm{N}\left(\overline{\mathrm{y}}_{\mathrm{k}}\right)$

$\mathrm{V}(\hat{\mathrm{Y}})=\mathrm{N}^{2} \mathrm{~V}\left(\overline{\mathrm{y}}_{\mathrm{k}}\right)$

$\hat{\mathrm{V}}(\hat{\mathrm{Y}})=\mathrm{N}^{2} \hat{\mathrm{V}}\left(\overline{\mathrm{y}}_{\mathrm{k}}\right)$

where $\hat{\mathrm{V}}\left(\overline{\mathrm{y}}_{\mathrm{k}}\right)=(\mathrm{N}-\mathrm{n}) /(\mathrm{nN}) \sum_{\mathrm{k}=1}^{\mathrm{n}}\left(\mathrm{y}_{\mathrm{k}}-\overline{\mathrm{y}}_{\mathrm{k}}\right)^{2} /(\mathrm{n}-1)$.

\section{Binomial model}

Another approach toward estimating $\mathrm{V}(\mathrm{Y})$ is to use a mathematical model which describes the subsampling process. Three assumptions are necessary to apply Ricker's (1937) binomial model: 1) each animal in a fraction to be divided has an equal probability of going into any of the I compartments of a subsampler, 2) each animal's outcome is independent of all others, and 3) each use of the subsampler is independent of previous uses. Under these conditions, $\mathrm{V}(\hat{\mathrm{Y}})$ may be calculated from a single count, $y_{k}$, of animals representing the $p_{k}$-th fraction of the sample:

$\mathrm{V}(\hat{\mathrm{Y}})=\mathrm{N}^{2} \mathrm{~V}\left(\mathrm{y}_{\mathrm{k}}\right)$

where $V\left(y_{k}\right)$ and its estimate $\hat{V}\left(y_{k}\right)$ are calculated from the binomial:

$\mathrm{V}\left(\mathrm{y}_{\mathrm{k}}\right)=\mathrm{YE}\left(\mathrm{p}_{\mathrm{k}}\right)\left[1-\mathrm{E}\left(\mathrm{p}_{\mathrm{k}}\right)\right]$

$\hat{\mathrm{V}}\left(\mathrm{y}_{\mathrm{k}}\right)=\mathrm{y}_{\mathrm{k}}\left[1-\mathrm{E}\left(\mathrm{p}_{\mathrm{k}}\right)\right]$.

For a Folsom plankton splitter with two equalsized compartments the general model may be simplified. Let $s$ be the number of sequential uses of the splitter to generate subsamples of fraction $1 / 2^{\mathrm{s}}$. Then

$$
\begin{aligned}
& \hat{Y}=2^{s} y_{k} \\
& V(\bar{Y})=Y\left(2^{s_{-}}-1\right) \\
& \hat{V}(\bar{Y})=2^{s} y_{k}\left(2^{s_{-}}-1\right) .
\end{aligned}
$$


This model may be used to calculate the coefficient of variation of $\hat{Y}$ :

$\mathrm{CV}(\hat{\mathrm{Y}})=100\left[\mathrm{Y}\left(2^{\mathrm{s}}-1\right)\right]^{1 / 2} / \hat{\mathrm{Y}}$

and its estimate

$\mathrm{CV}(\hat{\mathrm{Y}})=100\left[2^{\mathrm{s}} \mathrm{y}_{\mathrm{k}}\left(2^{\mathrm{s}}-1\right)\right]^{1 / 2} /\left(2^{\mathrm{s}} \mathrm{y}_{\mathrm{k}}\right)$.

$\mathrm{CV}(\hat{\mathrm{Y}})$ is given as a fucntion of $\mathrm{y}_{\mathrm{k}}$ and $\mathrm{s}$ in Fig. 2. Note that for large s a Poisson model is appropriate and

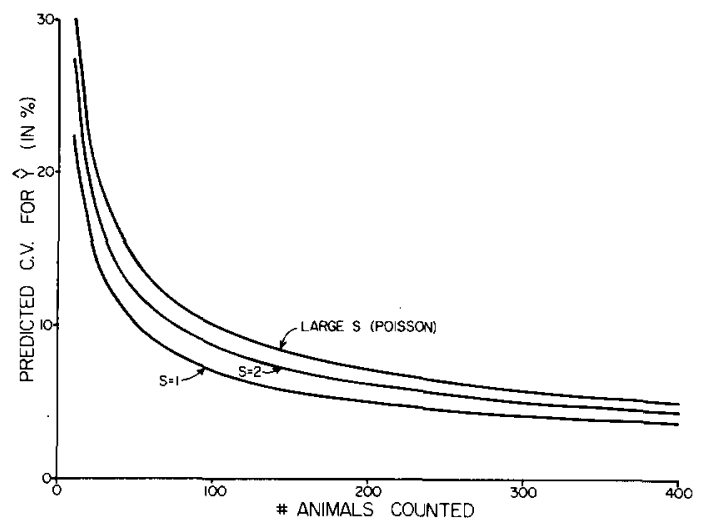

Fig. 2. The predicted coefficient of variation for the estimate of total number of animals in a sample $(\mathrm{CV}(\hat{\mathrm{Y}}))$ as a function of the number of animals counted in a subsample $\left(y_{k}\right)$ derived from the binomial subsampling model. $s$ is the number of sequential uses of the plankton splitter.
$\mathrm{CV}(\hat{\mathrm{Y}}) \doteq 100\left(\mathrm{y}_{\mathrm{k}}\right)^{1 / 2} / \mathrm{y}_{\mathrm{k}}$

Use of the Folsom plankton splitter for Great Lakes zooplankton

Coefficients of variation ( $\mathrm{CV}$ ) calculated from replicate subsamples of the 14 special hauls are shown in Fig. 3 versus the mean number of animals counted. Because a high degree of subsampling was

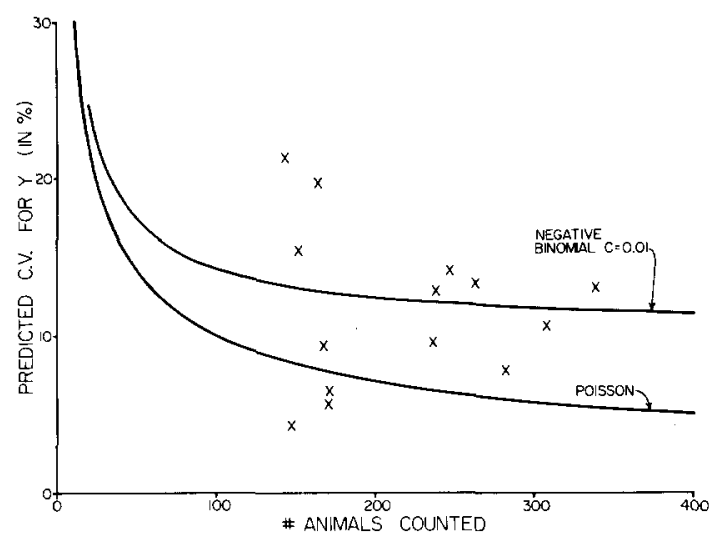

Fig. 3. Calculated coefficient of variation for the estimate of total number of animals in a sample $(\mathrm{CV}(\overline{\mathrm{Y}}))$ as a function of the mean number of animals counted in a subsample $\left(\bar{y}_{k}\right)$. Curves representing expectations from the Poisson and negative binomial (with clumping parameter $\mathrm{c}=0.01$ ) formulations are also shown.

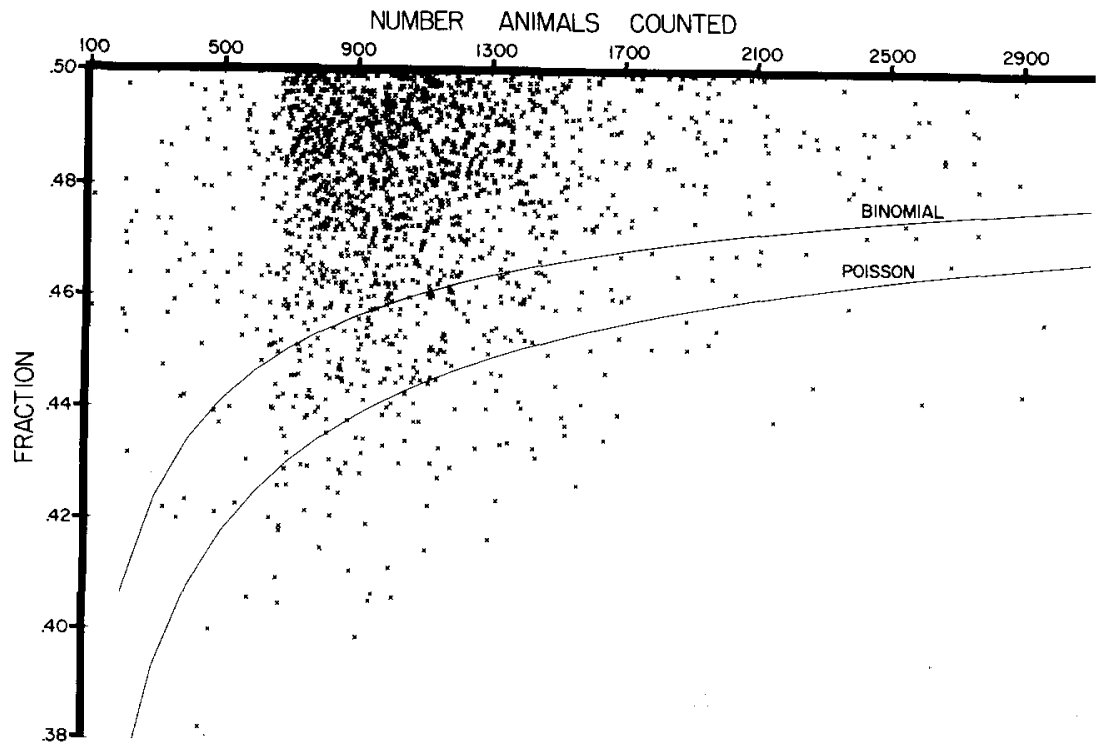

Fig. 4. The fraction of animals in the smaller of paired splits versus the total number of animals in both compartments. The $99 \%$ confidence limits, derived from the binomial and Poisson distributions, are also shown. 
performed, the appropriate random expectation is derived from the Poisson model. Also shown in Fig. 3 is the expected CV for a negative binomial distribution with a clumping parameter value (Cassie 1971) of $c=0.01$. In spite of the small sample size, it appears that the observed CVs tended to be greater than expected from the Poisson model.

A more substantial data set on which to test this observation consists of counts of zooplankton in pairs of splits from 1791 samples collected from nearshore Lake Michigan between April 1974 and June 1979. For each pair of splits the fraction of animals in the smallest split is plotted in Fig. 4 against the total number of animals in both compartments. Because the paired splits represent the outcome of a single use of the Folsom splitter, the binomial model is the appropriate random model. However, if the data are more variable than expected from the Poisson model then there must be an even stronger departure from the binomial. The 99\% confidence limits, calculated using normal approximations for binomial and Poisson distributions, are also shown. Approximately $15 \%$ of the points lie outside the binomial $99 \%$ confidence limit and $5 \%$ lie outside the poisson confidence limits. A direct check of the fit to a Poisson model for the plankton splitter consists of comparing the mean to the variance (Winsor \& Walford 1936; McEwen et al. 1954; Cassie 1971; Elliott 1971):

$\chi^{2}=\left(y_{1}-y_{2}\right)^{2} /\left(y_{1}+y_{2}\right)$.

If the distribution of splits truly followed the Poisson distribution, the test statistic should approximate $\chi_{1}^{2}$. Table 1 gives the sum of values for each date and its degrees of freedom (the number of pairs of splits). At all but four dates the statistic is significantly $(\mathrm{p}<0.05)$ larger than expected from a Poisson distribution.

\section{Comparison of sampling and subsampling variabil-} ity

The variance components estimated for sampling and subsampling in the June 1980 collections using the ANOVA model are given in Table 2. Variability between hauls accounted for a larger fraction of the total variance than did variability between subsamples for either the $78-\mu \mathrm{m}$ or the $156-\mu \mathrm{m}$ mesh net collections. The estimated coefficient of variation for hauls (ignoring subsampling variability) was
Table 1. Degrees of freedom, chi-square values for the comparison of mean to variance, and their significance for deviations of replicate subsamples by month of collection. ${ }^{*}$ indicates $\mathrm{p}<$ 0.05 , ns indicates $\mathrm{p}>0.05$.

\begin{tabular}{|c|c|c|c|}
\hline Date & D.F. & $\chi^{2}$ & Signif. \\
\hline $4 / 74$ & 51 & 116.6 & $*$ \\
\hline $5 / 74$ & 25 & 81.5 & $*$ \\
\hline $6 / 74$ & 24 & 75.1 & $*$ \\
\hline $7 / 74$ & 45 & 250.0 & $*$ \\
\hline $8 / 74$ & 23 & 92.5 & $*$ \\
\hline $9 / 74$ & 26 & 95.7 & $*$ \\
\hline $10 / 74$ & 53 & 165.0 & $*$ \\
\hline $4 / 75$ & 59 & 210.3 & $*$ \\
\hline $5 / 75$ & 27 & 78.2 & $*$ \\
\hline $6 / 75$ & 26 & 98.2 & $*$ \\
\hline $7 / 75$ & 54 & 279.2 & $*$ \\
\hline $8 / 75$ & 59 & 193.1 & $*$ \\
\hline $9 / 75$ & 28 & 109.7 & $*$ \\
\hline $10 / 75$ & 54 & 257.6 & $*$ \\
\hline $12 / 75$ & 30 & 177.2 & * \\
\hline $4 / 76$ & 49 & 258.0 & $*$ \\
\hline $5 / 76$ & 52 & 172.5 & $*$ \\
\hline $6 / 76$ & 28 & 108.6 & $*$ \\
\hline $7 / 76$ & 56 & 131.9 & $*$ \\
\hline $8 / 76$ & 28 & 71.2 & $*$ \\
\hline $9 / 76$ & 28 & 65.4 & $*$ \\
\hline $10 / 76$ & 52 & 198.6 & $*$ \\
\hline $4 / 77$ & 60 & 225.0 & $*$ \\
\hline $5 / 77$ & 28 & 33.6 & $\mathrm{~ns}$ \\
\hline $6 / 77$ & 28 & 85.5 & $*$ \\
\hline $7 / 77$ & 60 & 231.8 & * \\
\hline $8 / 77$ & 28 & 131.0 & * \\
\hline $9 / 77$ & 28 & 78.1 & * \\
\hline $10 / 77$ & 60 & 138.6 & $*$ \\
\hline $11 / 77$ & 28 & 39.8 & ns \\
\hline $12 / 77$ & 28 & 70.8 & $*$ \\
\hline $4 / 78$ & 60 & 113.4 & $*$ \\
\hline $5 / 78$ & 28 & 41.7 & ns \\
\hline $6 / 78$ & 28 & 105.4 & $*$ \\
\hline $7 / 78$ & 60 & 193.1 & * \\
\hline $8 / 78$ & 28 & 41.5 & ns \\
\hline $9 / 78$ & 28 & 94.5 & $*$ \\
\hline $10 / 78$ & 60 & 117.2 & * \\
\hline $11 / 78$ & 28 & 124.0 & * \\
\hline $4 / 79$ & 55 & 163.2 & * \\
\hline $5 / 79$ & 24 & 71.1 & $*$ \\
\hline $6 / 79$ & 26 & 72.0 & * \\
\hline
\end{tabular}

Table 2. Estimates of variance components (as \% of total variance), based on a random effects ANOVA model, due to subsampling and collecting replicate net hauls for nets having $156 \mu \mathrm{m}$ and $78 \mu \mathrm{m}$ mesh netting.

\begin{tabular}{lcc}
\hline Variance component & $156 \mu \mathrm{m}$ & $78 \mu \mathrm{m}$ \\
\hline Variability from hauls & $54 \%$ & $67 \%$ \\
Variability from subsamples & $46 \%$ & $33 \%$ \\
\hline
\end{tabular}


$12.8 \%$ for the $156-\mu \mathrm{m}$ mesh net and $19.8 \%$ for the 78- $\mu \mathrm{m}$ mesh net.

An optimal allocation of effort may be derived from the ANOVA model based on the ratio of costs of collecting samples compared to enumerating subsamples and the ratio of variance components attributed to these two sampling phases (Sokal \& Rohlf 1969). Figure 5 presents in graphical form the optimal number of subsamples (rounded to the nearest integer value) to be examined from each sample determined in this manner. Assuming the total combined cost of these sampling phases is fixed, the more subsamples examined from each sample, the fewer samples can be collected. The range of the two variance ratios presented in Table 2 and an hypothetical range of cost ratios appropriate for our study are indicated in Fig. 5. The resulting region defines a desired range of subsamples examined per sample to be from one to three.

\section{Discussion}

The most often used criteria for 'randomness' are based on the Poisson distribution (Cassie 1971;

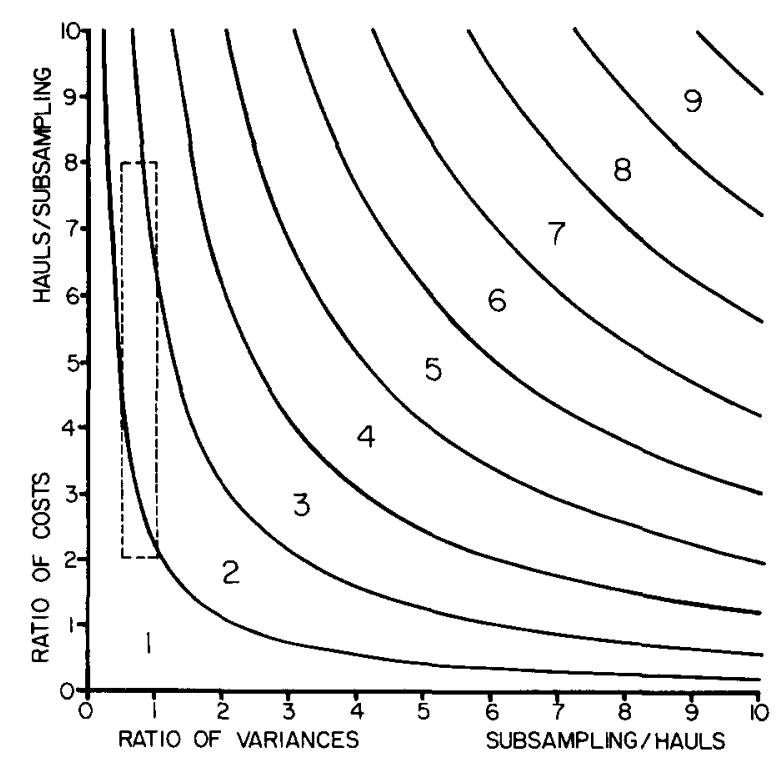

Fig. 5. The optimal number of subsamples (rounded to the nearest integer) to be examined from a sample, determined from the ratio of cost of collecting a net haul compared to the cost of examining a subsample, and the ratio of variances attributed to subsampling and net hauls. This formulation is derived from an ANOVA model.
Elliott 1971) whether or not there is a straightforward interpretation of its use. Poisson models of randomness have allowed insight into subsampling schemes for chambered devices, such as the Sedgewich-Rafter cell, which are composed of a large number of fields (Serfling 1949; Kutkuhn 1958). As shown above, the binomial subsampling model has a general application in describing the operation of compartment-type subsamplers, with the Poisson model appropriate when small fractions of the original sample are examined. The binomial model allows an intuitive interpretation of randomness of subsampling for compartment-type devices in that each individual organism is viewed as having an equal chance of settling into any compartment. Although graphical analyses (e.g. Fig. 4) are apt, one advantage of applying the Poisson model is that the chi-square test for equality of mean and variance is easy to perform.

The finding that the Folsom plankton splitter produced greater variability than expected from a random model may be explained in two ways: 1 ) if assumption \#1 of the binomial model were violated such that organisms had an unequal chance of settling into the two compartments, a larger variance than predicted would result; 2) zooplankton appendages become entwined and cladoceran carapaces are notorious for their tendency to adhere to one another despite precautions to disperse them these factors violate assumption $\# 2$ underlying the binomial model that each animal's outcome is independent of all others. Our frequent inspection of the Folsom apparatus indicated that differences in amounts of water divided between compartments were small. On this basis, it appears that the first assumption of the binomial model was not violated seriously. McEwen et al. (1954) and Longhurst \& Seibert (1967) discussed clumping of particular marine plankton organisms when using the Folsom splitter and both studies suggested that clumping was partially responsible for higher than expected variances.

The large sample size employed in this study potentially allows the detection of relatively small departures from randomness. Many published examinations of subsampling devices either have not used statistical tests or else have been limited to small sample sizes. Most studies employing statistical tests have concluded that compartment-type devices provide satisfactory results (Kott 1953; 
McEwen et al. 1954; Scarola \& Novotny 1968; Hickley 1975) although Longhurst \& Seibert (1967) cautioned against too ready an acceptance of previous statistical evaluations of Folsom subsampler performance.

The estimation of variance components and optimal numbers of subsamples to examine from each sample illustrates that, despite statistically significant departures from randomness, relatively few subsamples per sample needed to be worked up in the Lake Michigan study in order to obtain precise density estimates at a sampling location. Variability introduced by several sampling phases may be expected to differ with sampling design, study site, sampling gear (Table 2), and other factors such that a comprehensive analysis of optimal allocation of effort may not be possible. Indeed, it is likely that a fixed optimum effort does not strictly apply throughout the course of any study. Nevertheless, these statistical tools provide insight into experimental design that can not be obtained otherwise. These procedures are particularly useful in pilot studies but also have value when used in retrospect to evaluate techniques.

The focus of this paper has been on the use of subsamplers to estimate zooplankton population abundances from large initial samples. Vertical tows illustrate the advantage of collecting large samples in many studies because they integrate vertically stratified distributions of organisms. Subsampling of integrated collections allows precise estimates of average volumetric $\left(\# / \mathrm{m}^{3}\right)$ or areal $\left(\# / \mathrm{m}^{2}\right)$ densities. On the other hand, estimates of plankton spatial variability, or 'patchiness,' are most precise when small initial samples are obtained and subsampling is minimized (Venrick 1971). The particular objectives of a study and the nature of the variability of organisms within the habitat has the most direct influence on survey design (Cassie 1971; Venrick 1978; Cuff \& Coleman 1979; Green 1979), but variability introduced by subsampling is an important consideration.

\section{Acknowledgments}

This research was supported by the Indiana \& Michigan Electric Company. We thank Thomas D. Berry and Drs. William Y. B. Chang and Russell Moll for comments and criticisms of the manu- script. Stephen Wineberg provided helpful comments on the application of the binomial distribution and Dr. Alvin L. Jensen stimulated the initial interest in this study. Great Lakes Research Division Contribution No. 324.

\section{References}

Cassie, R., 1971. Statistics and sampling. In: Edmondson, W. T. \& G. C. Winberg (Eds.) A Manual on Methods for the Assessment of Secondary Productivity in Freshwaters. I.B.P. Handbook No. 17, Blackwell, Oxford. 358 pp.

Cuff, W. \& Coleman, N., 1979. Optimal survey design: lessons from a stratified random sample of macrobenthos. J. Fish. Res. Bd Can. 36: 351-361.

Cushing, C. E., 1961. A plankton sub-sampler. Limnol. Oceanogr. 6: $489-490$.

Elliott, J. M., 1971. Statistical analysis of samples of benthic invertebrates. Freshwater Biological Association Scientific Publication No. 25.

Evans, M. S., 1975. The 1974 preoperational zooplankton investigations relative to the Donald $\mathrm{C}$. Cook nuclear power plant. Spec. Resp. 58, Great Lakes Research Division, University of Michigan, Ann Arbor, Michigan.

Evans, M.S., Hawkins, B. E. \& Sell, D. W., 1980. Seasonal features of zooplankton assemblages of southeastern Lake Michigan. J. Great Lakes Res. 6: 275-289.

Evans, M. S., Wurster, T. E. \& Hawkins, B. E., 1978. The 1975 and 1976 operational zooplankton investigations relative to the Donald C. Cook nuclear power plant, with tests for plant effects (1971-1976). Spec. Rep. 64, Great Lakes Research Division, University of Michigan, Ann Arbor, Michigan.

Gibbons, S. C., 1933. A new type of sampler for use in the quantitative analysis of plankton collections. J. Cons. Perm. int. Explor. Mer 8: 195-200.

Green, R. H., 1979. Sampling Design and Statistical Methods for Environmental Biologists. John Wiley, New York. 257 pp.

Hawkins, B. E. \& Evans, M. S., 1979. Seasonal cycles of zooplankton biomass in southeastern Lake Michigan. J. Great Lakes Res. 5: 256-263.

Hickley, P, 1975. An apparatus for subdividing benthos samples. Oikos 26: 92-96.

Kott, P., 1953. Modified whirling apparatus for the subsampling of plankton. Aust. J. Mar. freshwat. Res. 4: 387-393.

Kutkuhn, J. H., 1958. Notes on the precision of numerical and volumetric plankton estimates from small-sample concentrates. Limnol. Oceanogr. 3: 69-83.

Longhurst, A. R. \& Seibert, D. L. R., 1967. Skill in the use of Folsom's plankton sample splitter. Limnol. Oceanogr. 12: 334-335.

McEwen, G. F., Johnson, M. W. \& Folsom, T. R., 1954. A statistical analysis of the performance of the Folsom plankton splitter, based upon test observations. Arch. Met. Geophys. Bioklim., Ser. A, 6: 502-527.

McWilliam, P.S., 1970. Seasonal changes in abundance and reproduction in the 'opossum shrimp', Mysis relicta Loven, in Lake Michigan. Masters thesis, Univ. of Sydney. 
Mednikov, B. M. \& Starbogatov, Y. A., 1961. The randomchamber for counting small biological objects. Trudy VGBO, Il (in Russian).

Motoda, S., 1959. Devices of simple plankton apparatus. Mem. Fac. Fish. Hokkaido Univ. 7: 73-94.

Raj, D., 1968. Sampling Theory. McGraw-Hill, New York. $302 \mathrm{pp}$.

Ricker, W. E., 1937. Statistical treatment of sampling processes useful in the enumeration of plankton organisms. Arch. Hydrobiol. 31: 68-84.

Scarola, J. F. \& Novotny, A. J., 1968. Folsom plankton splitter modified for enumeration of Entomostraca. Limnol. Oceanogr. 13: 195-196.

Serfling, R. E., 1949. Quantitative estimation of plankton from small samples of Sedgewick-Rafter-cell amounts of concentrate samples. Trans. Am. microsc. Soc. 68: 185-199.

Sokal, R. R. \& Rohlf, F. J., 1969. Biometry; the Principles and Practice of Statistics in Biological Research. W. H. Freeman, San Francisco. 776 pp.
Sukhatme, P. V.\& Sukhatme, B. V., 1970. Sampling Theory of Surveys with Applications, 2 nd edn. Iowa State Univ. Press, Ames. 452 pp.

Venrick, E. L., 1971. The statistics of subsampling. Limnol. Oceanogr. 16: 811-818.

Venrick, E. L., 1978. Systematic sampling in a planktonic ecosystem. Fishery Bull. 76: 617-627.

Waters, T. F., 1969. Subsampler for dividing large samples of stream invertebrate drift. Limnol. Oceanogr. 14: 813-815.

Wiborg, K. F., 1951. The whirling vessel. Rep. Norw. Fish. mar. Invest. 9: 1-16.

Winsor, C. P. \& Walford, L. A., 1936. Sampling variations in the use of plankton nets. J. Cons. perm. int. Explor. Mer 6: 190-204.

Received 6 October 1981; in revised form 24 November 1981. 